\title{
Correction to: TIP60 recruits SUV39H1 to chromatin to maintain heterochromatin genome stability and resist hydrogen peroxide-induced cytotoxicity
}

\author{
Bo Tu ${ }^{1,2,3} \cdot$ Yantao Bao ${ }^{1} \cdot$ Ming Tang $^{3} \cdot$ Qian $\mathrm{Zhu}^{1} \cdot$ Xiaopeng $\mathrm{Lu}^{1} \cdot$ Hui Wang ${ }^{1} \cdot$ Tianyun $\mathrm{Hou}^{3} \cdot$ Ying Zhao $^{3}$. \\ Ping Zhang ${ }^{3} \cdot$ Wei-Guo Zhu ${ }^{1,3,4,5}$
}

Published online: 20 November 2020

(c) Shenzhen University School of Medicine; Fondazione Istituto FIRC di Oncologia Molecolare 2020

\section{Correction to: Genome Instability \& Disease https://doi.org/10.1007/s42764-020-00025-8}

The original version of this article, published on November 6, 2020, contained a mistake.

The list of corresponding authors was incorrect. The correct list is given below. The original article has been corrected.

The original article can be found online at https://doi.org/10.1007/ s42764-020-00025-8.

Ping Zhang

zhangping332@bjmu.edu.cn

$\bowtie$ Wei-Guo Zhu

zhuweiguo@szu.edu.cn

1 Guangdong Key Laboratory of Genome Instability and Human Disease Prevention, Department of Biochemistry and Molecular Biology, Shenzhen University School of Medicine, Shenzhen 518055, China

2 Fred Hutchinson Cancer Research Center, Seattle, WA 98101, USA

3 Key Laboratory of Carcinogenesis and Translational Research (Ministry of Education), Beijing Key Laboratory of Protein Posttranslational Modifications and Cell Function, Department of Biochemistry and Molecular Biology, School of Basic Medical Sciences, Peking University Health Science Center, Beijing 100191, China

4 International Cancer Center, Shenzhen University School of Medicine, Room 901, Building A6, No. 1066, Xueyuan Avenue, Nanshan District, Shenzhen 518055, Guangdong, China

5 Shenzhen Bay Laboratory, Shenzhen University School of Medicine, Shenzhen 518055, China 